\title{
COMPARAÇÃO DO DESEMPENHO DE ATIVIDADES FUNCIONAIS EM CRIANÇAS COM DESENVOLVIMENTO NORMAL E CRIANÇAS COM PARALISIA CEREBRAL
}

\author{
Marisa C. Mancini', Patrícia M. Fiúza², Jerusa M. Rebelo², Lívia C. Magalhães³, \\ Zélia A. C. Coelho4, Maria Lúcia Paixão5, Ana Paula B. Gontijo', Sérgio T. Fonseca ${ }^{7}$
}

\begin{abstract}
RESUMO - Objetivo: Comparar o desempenho de atividades funcionais de auto-cuidado em crianças com desenvolvimento normal e crianças com paralisia cerebral (PC), utilizando o teste PEDI. Método: 142 crianças com desenvolvimento normal e 33 crianças com PC foram avaliadas em 22 itens da escala de auto-cuidado do teste funcional PEDI. A metodologia Rasch transformou os escores em incrementos de dificuldade intervalar de 0 a 100 (logit). 0 índice de correlação Spearman rank comparou a ordem dos logits nos dois grupos. Resultados: Onze itens apresentaram diferença significativa nos valores logit dos dois grupos. Destes, sete itens apresentaram dificuldade relativa superior no grupo de crianças com PC e quatro itens apresentaram dificuldade relativa maior no grupo de crianças normais. Foi observada ainda relação significativa na ordem dos 22 itens nas duas escalas intervalares. Conclusão: Resultados sugerem que o desenvolvimento de atividades funcionais de auto-cuidado pode ser influenciado pela presença de PC. Tais resultados podem subsidiar estratégias de avaliação e intervenção para crianças com distúrbios neuromotores.
\end{abstract}

PALAVRAS-CHAVE: função, paralisia cerebral, desenvolvimento normal, auto-cuidado.

\section{Comparison of functional activity performance in normally developing children and children with cerebral palsy}

\begin{abstract}
Objective: To compare the pattern of self-care performance in normal children and children with cerebral palsy (CP). Method: 142 normal children and 33 children with CP were evaluated by 22 items from the self-care scale of the PEDI functional test. Rasch methodology transformed scores into interval measures of difficulty from 0 to 100 (logit). Spearman rank correlation coefficient compared the order of logits in the two groups. Results: Eleven items showed significant differences in the logit values received. Out of these, 7 items showed relative difficulty values greater in the group of children with CP and 4 items showed relative difficulty values greater among normal children. A significant correlation was observed in the order of the 22 items displayed in the two interval scales. Conclusion: The results suggest that the development of self-care functional activities may be influenced by the presence of CP. These results may support assessment and intervention strategies for children with neuromotor disorders.
\end{abstract}

KEY WORDS: function, cerebral palsy, normal development, self-care.

A paralisia cerebral, também denominada encefalopatia crônica não progressiva da infância, é consequência de uma lesão estática, ocorrida no período pré, peri ou pós-natal que afeta o sistema nervoso central em fase de maturação estrutural e funcional. É uma disfunção predominantemente sensoriomotora, envolvendo distúrbios no tônus muscular, postura e movimentação voluntária' ${ }^{1}$ Estes distúrbios se caracterizam pela falta de controle sobre os movimentos, por modificações adaptativas do comprimento muscular e em alguns casos, chegando a resultar em deformidades ósseas ${ }^{2}$. Esta doença ocorre no período em que a criança apresenta ritmo acelerado de desenvolvimento, podendo comprometer

Universidade Federal de Minas Gerais (UFMG), Belo Horizonte MG, Brasil: 'Doutora em Ciências (Sc.D.), Professora Adjunta do Departamento de Terapia Ocupacional, Escola de Educação Física, Fisioterapia e Terapia Ocupacional (EEFFTO); ${ }^{2}$ Terapeuta Ocupacional, Bolsista de Iniciação Científica do Conselho Nacional de Desenvolvimento Científico e Tecnológico (CNPq); ${ }^{3}$ Doutora em Educação (Ph.D.), Professora Adjunta do Departamento de Terapia Ocupacional, EEFFTO; ${ }^{4}$ Especialista, Professora Auxiliar do Departamento de Terapia Ocupacional, EEFFTO; ${ }^{5}$ Especialista, Professora Assistente do Departamento de Fisioterapia, EEFFTO; ${ }^{6}$ Especialista, Professora Auxiliar do Departamento de Fisioterapia, EEFFTO; ${ }^{7}$ Doutor em Ciências (Sc.D.), Prof. Adjunto do Departamento de Fisioterapia, EEFFT. Este estudo foi financiado por: Conselho Nacional de Desenvolvimento Científico e Tecnológico (CNPq), pela Fundação de Amparo à Pesquisa do Estado de Minas Gerais (FAPEMIG) e Pró-Reitoria de Pesquisa da Universidade Federal de Minas Gerais.

Recebido 16 Outubro 2001, recebido na forma final 4 Janeiro 2002. Aceito 25 Janeiro 2002. 
o processo de aquisição de habilidades ${ }^{3}$. Tal comprometimento pode interferir na função, dificultando o desempenho de atividades frequentemente realizadas por crianças com desenvolvimento normal ${ }^{4}$.

Em países desenvolvidos observou-se um aumento nos casos de paralisia cerebral nas duas últimas décadas, com índices de prevalência dos casos moderados e severos variando entre 1,5 e 2,5 por 1.000 nascimentos ${ }^{5-8}$. Estes dados têm sido atribuídos à melhoria dos cuidados médicos perinatais, contribuindo para aumento da sobrevivência de crianças com idade gestacional e baixo peso ao nascimento cada vez mais extremos. Entre os recém-nascidos prétermo com muito baixo peso (inferior a $1500 \mathrm{~g}$ ) a presença de disfunções neurológicas é observada com maior frequência do que em crianças nascidas a termo com peso adequado, podendo a paralisia cerebral acontecer com frequência de 25 a 30 vezes mais no grupo de crianças consideradas de risco perinatal ${ }^{7}$. Em países subdesenvolvidos a incidência desta doença é maior do que nos países desenvolvidos, observando-se índices de 7:10007. No Brasil os dados estimam cerca de 30000 a 40000 casos novos por ano 9 .

Com base no modelo de classificação proposto pela Organização Mundial de Saúde ${ }^{1}$ esta enfermidade pode apresentar consequências variadas. No que se refere à função de órgãos e sistemas, a paralisia cerebral geralmente interfere no funcionamento do sistema músculo-esquelético. Neste nível, as características associadas a esta patologia incluem distúrbios de tônus muscular, postura e movimentação voluntária'. O comprometimento neuromotor desta doença pode envolver partes distintas do corpo, resultando em classificações topográficas específicas (e.g., quadriplegia, hemiplegia e diplegia) ${ }^{6,10}$. Outro tipo de classificação é a baseada nas alterações clínicas do tono muscular e no tipo de desordem do movimento podendo produzir o tipo espástico, discinético ou atetóide, atáxico, hipotônico e misto 6 . A gravidade do comprometimento neuromotor de uma criança com paralisia cerebral pode ser caracterizada como leve, moderada ou severa, baseada no meio de locomoção da criança ${ }^{10,11}$. Estas classificações servem a um propósito de descrição e caracterização da lesão, não fornecendo informação sobre as consequências desta enfermidade na rotina diária da criança.

Além das deficiências neuromotoras, a paralisia cerebral pode também resultar em incapacidades, ou seja, limitações no desempenho de atividades e tarefas do cotidiano da criança e de sua família'. Estas tarefas incluem, por exemplo, atividades de auto-cuidado como conseguir alimentar-se sozinho, tomar banho e vestir-se, ou atividades de mobilidade como ser capaz de levantar da cama pela manhã e ir ao banheiro, jogar bola e andar de bicicleta com amigos, além das atividades de características sociais e cognitivas como brincar com brinquedos e com outras crianças e freqüentar a escola. Informações sobre o desempenho de atividades funcionais como estas são extremamente relevantes, uma vez que as dificuldades no desempenho das mesmas constituem, geralmente, a queixa principal de crianças, pais e familiares. Portanto, a promoção do desempenho de atividades e tarefas funcionais pode ser definida como objetivo a ser alcançado pelas terapêuticas empregadas ${ }^{12,13}$.

O objetivo deste estudo foi descrever o padrão de desenvolvimento das habilidades funcionais de auto-cuidado em crianças com desenvolvimento normal e crianças com paralisia cerebral, comparando a ordem e as dificuldades relativas no desempenho de atividades funcionais de alimentação, banho, vestir e higiene pessoal, nestes dois grupos de crianças, utilizando o método de avaliação PEDI ${ }^{14}$.

\section{MÉTODO}

\section{Participantes}

Este estudo transversal contou com a participação de crianças de duas amostragens distintas que foram selecionadas de forma não aleatória, com base em critérios de inclusão previamente determinados para cada grupo. Os critérios utilizados para inclusão de crianças no grupo normativo foram: apresentar desenvolvimento normal, ter nascido a termo, não fazer uso regular de medicamento e não apresentar problemas físicos, sensoriais ou emocionais. Para participação no grupo de paralisia cerebral os critérios de inclusão foram: ter diagnóstico definitivo desta doença e apresentar severidade leve ou moderada. Foram excluídas deste estudo crianças que apresentassem distúrbios associados (retardo mental, epilepsia, déficit sensorial). O critério para classificar a severidade das crianças com paralisia cerebral foi baseado no meio de locomoção ${ }^{11}$. Crianças que apresentavam locomoção independente foram classificadas como leve; aquelas que se locomoviam com algum auxílio (bengala, muleta, andador) foram identificadas como severidade moderada e as que não se locomoviam (e.g., faziam uso de cadeira de rodas) foram consideradas graves; crianças com severidade grave foram excluídas deste estudo.

Cada um dos grupos foi estratificado em relação à variável sexo, visando incluir um número equivalente de crianças de ambos os sexos.

\section{Instrumentação}

Crianças de ambos os grupos foram avaliadas pelo teste funcional norte americano Pediatric Evaluation of Disability 
Inventory (PEDI) ${ }^{14}$ que foi traduzido para o português ${ }^{15} \mathrm{e}$ adaptado para contemplar as especificidades sócio-culturais do Brasil, com permissão e colaboração dos autores da avaliação original. O PEDI ${ }^{14,15}$ é uma avaliação realizada através de entrevista com pais ou responsáveis que possam informar sobre o desempenho típico da criança em casa. Este teste avalia aspectos funcionais do desenvolvimento de crianças com idades entre 6 meses e 7 anos e meio, em três áreas de desempenho: auto-cuidado, mobilidade e função social. Este teste pode ser usado para crianças com idades superiores a 7 anos e meio, caso seu desenvolvimento funcional encontre-se dentro da faixa etária proposta ${ }^{14,15}$.

Esta avaliação é dividida em três partes distintas, sendo que cada parte inclui as três áreas de desempenho citadas acima. A primeira parte avalia as habilidades ou capacidades funcionais da criança; a segunda parte informa sobre a quantidade de ajuda ou assistência que a criança recebe para desempenhar as atividades funcionais e a terceira parte documenta as modificações do ambiente necessárias para o desempenho de tarefas funcionais.

Este estudo utilizou apenas a escala de auto-cuidado da parte de habilidades funcionais. Esta escala consiste em 73 itens agrupados em 5 tarefas básicas de auto-cuidado: alimentação, banho, vestir, higiene pessoal e uso do banheiro. Neste estudo foram investigadas as tarefas de alimentação, banho, vestir e higiene pessoal, totalizando 22 itens funcionais. Cada item é avaliado com escore 0 (zero) se a criança não for capaz de desempenhar a atividade ou 1 (um) se ela for capaz de desempenhar a atividade ou a mesma já fizer parte do seu repertório funcional. Cada escala do teste fornece um escore total que é o resultado da pontuação dos itens da mesma. O manual ${ }^{14}$ fornece critérios específicos para pontuação de cada item do teste.

\section{Procedimento}

As crianças dos dois grupos foram avaliadas por três examinadores previamente treinados na aplicação do teste PEDI. Após treinamento, a confiabilidade entre examinadores foi investigada pela consistência dos três examinadores na avaliação de dez crianças com desenvolvimento típico ou normal, obtendo-se índice elevado de coeficiente de correlação intra-classe (I.C.C. $=0,998$ ).

A avaliação das crianças consistiu de uma entrevista estruturada com pais ou responsáveis e foi realizada no período entre agosto de 1999 e junho de 2000. Antes de serem entrevistados, os pais assinaram termo de consentimento para participação da criança no estudo. Este estudo foi aprovado pelo Comitê de Ética em Pesquisa da UFMG em 16 de junho de 1999.

Todas as crianças que participaram deste estudo residiam na região metropolitana de Belo Horizonte. As entrevistas foram realizadas em creches, escolas públicas, escolas particulares, residências ou outro local de maior conveniência para os pais ou responsáveis pelas crianças.

\section{Análise estatística}

A metodologia Rasch foi usada para transformar os escores obtidos na avaliação das crianças. Rasch é o nome dado a um modelo de transformação logística que se baseia no pressuposto que mensurações devem ser baseadas em contínuos intervalares ${ }^{16}$. Esta metodologia realiza transformações matemáticas para converter dados de escala ordinal em medidas intervalares, dispondo-os em contínuo cuja unidade padrão é chamada de logit. As unidades logit deste contínuo transformado representam incrementos de dificuldade relativa ${ }^{16,17}$.

Neste estudo, a metodologia Rasch foi utilizada para desenvolvimento de dois contínuos lineares simples, divididos em intervalos iguais que caracterizam incrementos de dificuldade relativa numa escala de 0 a 100 . Quanto maior o valor do logit de determinado item, maior o nível de dificuldade daquele item em relação aos outros itens ${ }^{17}$. O primeiro contínuo ilustra a disposição das atividades funcionais desempenhadas por crianças do grupo normativo e o segundo contínuo informa sobre o desempenho de crianças portadoras de paralisia cerebral, nos mesmos itens funcionais. Diferenças nos valores logits dos itens funcionais em ambos os grupos foram analisadas por um método gráfico recomendado por Wright e Stone ${ }^{18}$. Em um gráfico simples, representou-se a calibração de itens da amostragem normativa versus da amostragem de crianças com paralisia cerebral; traçou-se linha de controle de qualidade usando os erros associados à estimativa de cada calibração ( $\pm 2 \mathrm{E}=95 \%$ do intervalo de confiança). A calibração média das duas amostragens é centrada no zero e a relação entre os itens deve ser estatisticamente linear, com a maioria dos itens localizados entre as linhas de controle de qualidade. Desta forma, atividades funcionais que apresentarem valores logit semelhantes nos dois grupos teriam um tempo de aquisição relativamente semelhante pelos dois tipos de crianças. Por outro lado, atividades funcionais que se localizarem fora das linhas de controle de qualidade teriam valores logit diferentes nos dois grupos. Segundo critério utilizado na literatura ${ }^{16}$ quando mais de $5 \%$ dos itens apresentarem dificuldade relativa diferente num grupo em relação ao outro, ou seja, localizarem fora das linhas de controle de qualidade, podese argumentar diferença na distribuição dos itens nos dois grupos. Esta informação foi utilizada para avaliar diferenças nos logits das atividades funcionais nos contínuos normativo e de crianças com paralisia cerebral.

O índice de correlação Spearman rank $\left(r_{s}\right)$ foi utilizado para comparar a ordem de disposição dos 22 itens ou atividades funcionais nos dois contínuos. Para todas as análises realizadas foi considerado nível de significância $\alpha=0,05$.

\section{RESULTADOS}

O primeiro grupo (normativo) foi constituído de 142 crianças que apresentavam desenvolvimento normal. A idade das crianças deste grupo variou de 
3 a 7,6 anos (média $=4,9$ anos; desvio padrão $=1,3$ anos). Neste grupo, metade dos participantes era do sexo feminino $(n=71)$ e a outra metade do sexo masculino $(n=71)$.

O segundo grupo foi composto por 33 crianças com diagnóstico de paralisia cerebral, sendo 18 do sexo feminino e 15 do sexo masculino. A idade das crianças variou de 3 a 8 anos de idade (média $=5,6$ anos; desvio padrão $=2,0$ anos). A maioria das crianças apresentou severidade leve $(n=25)$ e um menor número teve severidade classificada como moderada $(n=8)$, incluindo crianças com diagnósticos de hemiplegia $(n=15)$, diplegia $(n=13)$ e quadriplegia $(n=5)$.

Figura 1 mostra a disposição hierárquica dos 22 itens funcionais nos contínuos lineares, com base no desempenho de crianças normais e crianças com paralisia cerebral. O número entre parênteses localizado após cada item funcional indica o valor logit do item no grupo. Itens localizados na parte superior de cada contínuo apresentam uma dificuldade relativa maior e aqueles localizados na parte inferior têm dificuldade relativa menor para as crianças de um mesmo grupo. O índice de correlação Spearman rank que comparou a ordem de disposição das 22 atividades funcionais nos grupos normativo e de crianças com paralisia cerebral indicou uma associação forte e significante $\left(r_{s}=0,83 ; p<0,0001\right)$.

A Figura 2 mostra que dos 22 itens analisados, 11 apresentaram valor logit diferente nos dois contínuos (itens localizados fora das linhas de controle de qualidade traçadas no gráfico). Este número representa $50 \%$ do total de itens analisados, indicando diferença na dificuldade relativa destes itens nos contínuos lineares, segundo critério proposto pela literatura e definido anteriormente $(n>5 \%)$. Destes onze itens que apresentaram diferença no valor logit dos dois grupos, sete atividades funcionais apresentaram índice de dificuldade relativa (valor logit) superior no grupo de crianças com paralisia cere-

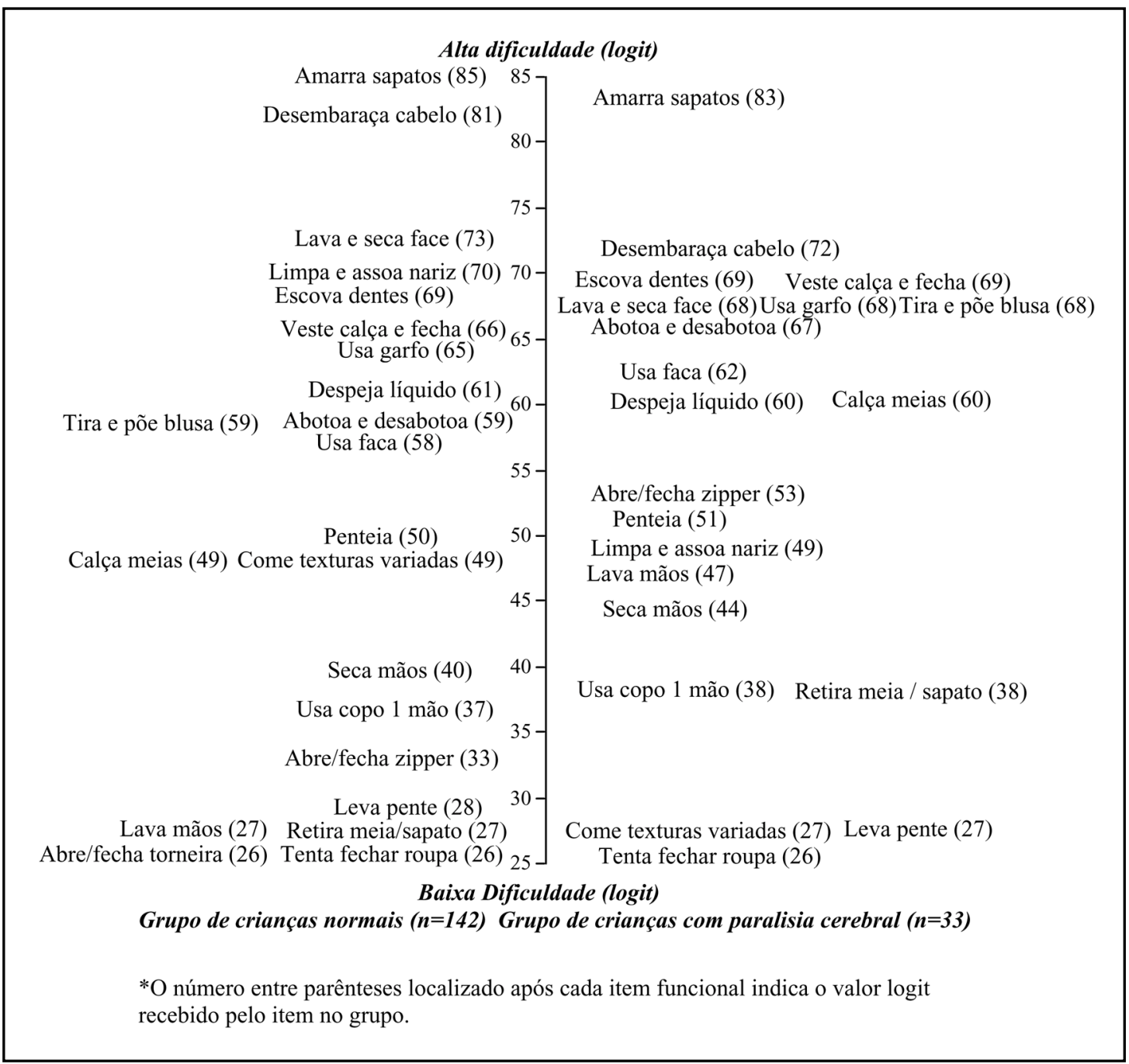

Fig 1. Disposição hierárquica dos itens funcionais em dois contínuos lineares, conforme nível de dificuldade (logit)*. 


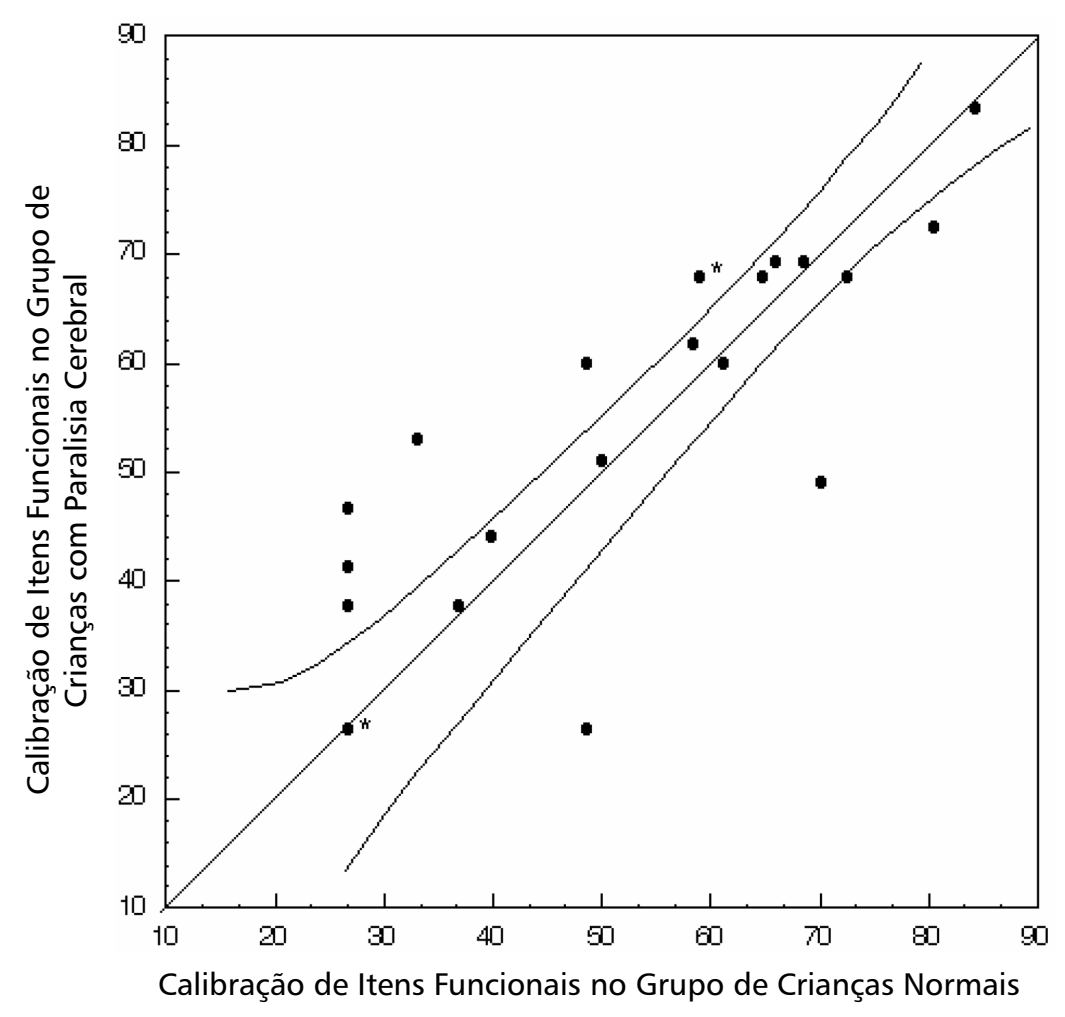

* Dois itens tiveram o mesmo valor de calibração logit em ambos os grupos e estão graficamente representados por um único ponto.

** Itens localizados sobre e fora das linhas de controle de qualidade traçadas no gráfico $(n=11)$ apresentaram diferença significativa no valor logit recebido nos dois grupos. O sentido da diferença é ilustrado pela localização dos itens fora das linhas de controle de qualidade. Sete itens apresentaram dificuldade relativa significativamente superior no grupo de crianças com paralisia cerebral (próximos ao eixo $Y$ ) e quatro itens tiveram valores logit significativamente superiores no grupo de crianças normais (próximos ao eixo $\mathrm{X}$ ). Os itens localizados dentro das linhas de controle de qualidade $(n=11)$ tiveram dificuldade relativa (logit) semelhante nos dois grupos de crianças.

Fig 2. Gráfico comparativo da calibração dos itens funcionais nos grupos de crianças normais e crianças com paralisia cerebral com linhas de controle de qualidade (Intervalo de Confiaça de 95\%)**

bral em relação à dificuldade das outras atividades (abrir e fechar a torneira, lavar as mãos, tirar e colocar blusa, abotoar e desabotoar, calçar e retirar meias, retirar sapatos e abrir e fechar zíper). Estes itens estão localizados fora das linhas de controle de qualidade e próximos à coordenada do grupo de crianças com paralisia cerebral (vertical). Os outros quatro itens que apresentaram diferença significativa tiveram valor logit maior no grupo normativo, comparado com o grupo de crianças com paralisia cerebral. Este número representa $18 \%$ dos itens avaliados neste estudo $(n=22)$ e descrevem habilidades como comer texturas variadas, desembaraçar cabelo, limpar e assoar nariz e lavar e secar a face. Estes itens também estão localizados fora das linhas de controle de qualidade e próximos à coordenada do grupo de crianças normais (horizontal).

\section{DISCUSSÃO}

Os resultados deste estudo fornecem informações importantes sobre o desempenho de atividades funcionais nos dois grupos de crianças. A ordem de disposição das habilidades funcionais de auto-cuidado nos dois grupos foi relativamente equivalente. Este resultado mostra que, mesmo na presença de distúrbios e limitações motoras, as crianças com paralisia cerebral tendem a desempenhar atividades funcionais de sua rotina diária em uma seqüência semeIhante à das crianças com desenvolvimento normal.

Esta similaridade na ordem de aquisições funcionais pode ser justificada por dois fatores. Como primeiro fator, a semelhança na ordem das habilidades pode refletir o efeito do processo maturacional no desempenho funcional, onde o desenvolvimento de componentes específicos de desempenho tais como massa corporal, força e coordenação poderiam influenciar progressivamente a realização de atividades funcionais, mesmo na presença de paralisia cerebral. Um segundo fator pode ser atribuído à expectativa de desempenho dos pais, que pode ter sido semelhante nos dois grupos, ilustrando assim uma influência do ambiente sobre o desempenho.

Apesar da relativa consistência na ordem de disposição das habilidades funcionais nos dois contínuos, algumas diferenças foram observadas. O grau de dificuldade das atividades funcionais nos dois grupos foi significativamente diferente em $50 \%$ dos itens avaliados. Este resultado indica que o mesmo 
item funcional apresenta um nível de complexidade diferenciado nos dois grupos, em relação à dificuldade dos demais itens no mesmo contínuo, representando um desafio maior para crianças com paralisia cerebral. Sete dos 22 itens avaliados apresentaram dificuldade relativamente maior no grupo de crianças com paralisia cerebral do que no grupo normativo. Estes itens descrevem capacidades funcionais da rotina da criança como abrir e fechar a torneira, lavar as mãos, tirar e colocar blusa, abotoar e desabotoar, calçar e retirar meias, retirar sapatos e abrir e fechar zíper. Uma característica comum observada entre os itens destacados é que eles ilustram atividades funcionais que requerem não só habilidades bimanuais como também controle postural e maior precisão da função manual. Dificuldades no desempenho destas atividades funcionais ilustram o impacto da paralisia cerebral na rotina diária destas crianças.

Os itens de habilidades de auto-cuidado tais, como comer texturas variadas, desembaraçar cabelo, limpar e assoar nariz e lavar e secar a face, apresentaram maior dificuldade relativa no grupo normativo comparado com o grupo de crianças com paralisia cerebral. É possível que tais resultados possam ser explicados pela influência de valores culturais no desenvolvimento infantil. A cultura brasileira tende a ser protecionista com relação às crianças na realização de tarefas da rotina diária ${ }^{19}$. Desta forma, embora a criança normal possa apresentar tais capacidades, elas muitas vezes não chegam a utilizálas em sua rotina diária. Por outro lado, pais de crianças com paralisia cerebral são frequentemente orientados pelos profissionais que as atendem a estimularem o uso de diferentes habilidades e a promoverem a sua independência e autonomia funcional.

Outro ponto que indicou diferença entre os dois grupos foi a disposição geral dos itens funcionais em cada contínuo. No grupo de crianças com paralisia cerebral essa disposição foi mais heterogênea, apresentando espaços e agrupamento de itens ao longo da escala intervalar enquanto no grupo de crianças com desenvolvimento normal os itens apresentaram-se dispostos hierarquicamente em pequenos incrementos de dificuldade ao longo do contínuo. Esta informação sugere que no grupo de crianças normais o desenvolvimento de habilidades funcionais tende a seguir uma certa linearidade gradativa e no grupo de crianças com paralisia cerebral essa sequência de aquisições tende a ser menos uniforme. É possível que as dificuldades de controle do movimento apresentadas pelas crianças com parali- sia cerebral influenciem no desempenho funcional das mesmas, diferenciando o padrão apresentado por elas daquele apresentado pelas crianças normais.

A literatura apresenta várias evidências sobre o desempenho funcional de crianças com desenvolvimento normal ${ }^{20-26}$. Estas informações vêm sendo tradicionalmente utilizadas para orientar o processo de avaliação e tratamento de crianças com distúrbios do desenvolvimento, incluindo paralisia cerebral ${ }^{25}$. Tal conduta se fundamenta no pressuposto clínico de que a sequência e o tempo das aquisições do desenvolvimento infantil possam ser semelhantes nestes dois grupos de crianças $^{27}$. Pressupostos clínicos como este precisam ser testados cientificamente para que a prática destes profissionais possa ser pautada em evidências.

Os dados deste estudo sugerem que existem tanto similaridades quanto diferenças no desempenho de atividades funcionais em crianças com desenvolvimento normal e com paralisia cerebral. A literatura nesta área ainda encontra-se bastante escassa e alguns autores abordam este tema de forma singular. Brown e Gordon ${ }^{28}$ investigaram o impacto da paralisia cerebral no repertório de atividades diárias destas crianças e verificaram que elas tendem a ser mais dependentes dos pais, desempenham menor variedade de atividades diárias com menor participação em atividades sociais e de recreação. Mackie et al. ${ }^{29}$ desenvolveram um instrumento para avaliar o impacto da deficiência e da incapacidade na vida de crianças com paralisia cerebral e de suas famílias e constataram que quanto maior a limitação funcional apresentada pela criança, maior é esse impacto. Lepage et al. ${ }^{4}$ investigaram a associação entre as características da locomoção e a realização de atividades da rotina diária em crianças com paralisia cerebral, mostrando que as habilidades de locomoção estão associadas com a realização de atividades diárias e sociais. Estes estudos apresentam evidências importantes sobre as consequências funcionais de doenças neurológicas na infância como a paralisia cerebral. Em geral, eles investigaram relação entre níveis diferentes do modelo de classificação proposto pela Organização Mundial da Saúde ${ }^{1}$ em um único grupo clínico (paralisia cerebral). O presente estudo comparou o desempenho de dois grupos distintos num mesmo nível funcional (atividade) ${ }^{1}$. Os dados apresentados pelo presente estudo adicionam informações a este crescente corpo de conhecimento.

O presente estudo possui duas limitações que devem ser identificadas. Em primeiro lugar, a amostra de crianças com paralisia cerebral foi bem menor do 
que a de crianças normais. Na verdade, esta diferença no número de crianças dos dois grupos reflete variações de tamanho observadas também nas duas populações infantis (crianças normais e crianças com paralisia cerebral). Em segundo lugar, o modelo de pesquisa utilizado neste estudo foi o transversal que restringe inferências sobre mudanças longitudinais no desenvolvimento infantil.

No sentido de fornecer um entendimento mais amplo sobre o impacto funcional da paralisia cerebral, é necessário que o desenvolvimento de habilidades funcionais em crianças com distúrbios neuromotores seja abordado de forma mais específica, buscando-se identificar os moderadores e mediadores deste processo. Este entendimento possibilitaria melhorar a atuação terapêutica dos diversos profissionais da equipe, a orientação aos familiares que convivem diariamente com as limitações destas crianças e o ensino de novos profissionais que chegam no mercado. Tais esforços devem ser estimulados não só em grupos clínicos como de crianças com paralisia cerebral, como também nas diferentes categorias que compõem este grupo clínico.

Concluindo, é na limitação funcional, ou seja, durante o desempenho de atividades e tarefas da rotina diária que a incapacidade da criança é manifestada ${ }^{12,13,28}$. Todas as argumentações feitas sobre as semelhanças e diferenças observadas nos dois contínuos apresentam-se como hipóteses a serem testadas por outras investigações científicas. Os resultados apresentados neste estudo contribuem para uma melhor compreensão do desenvolvimento humano em condições diferenciadas e fornecem subsídios para fundamentar estratégias de avaliação e intervenção.

Agradecimentos - Agradecemos, às seguintes instituições situadas em Belo Horizonte, MG, que colaboraram com este estudo: Ambulatório Bias Fortes do Complexo Hospital das Clínicas da UFMG, Hospital Municipal Odilon Behrens, Associação Mineira de Reabilitação (AMR) e Centro Geral de Reabilitação (CGR).

\section{REFERÊNCIAS}

1. World Health Organization. International classification of function and disability, Beta-2 Version. Geneva: WHO, 1999.

2. Shepherd RB. Fisioterapia em pediatria. 3.Ed. São Paulo: Santos Livraria Editora, 1996:110-144.

3. Wilson JM. Cerebral palsy. In Campbell SK (ed). Clinics in physical therapy: pediatric neurologic physical therapy. 2. Ed. New York: Churchill Livingstone, 1991:301-360.
4. Lepage C, Noreau L, Bernard P. Association between characteristics of locomotion and accomplishment of life habits in children with cerebral palsy. Phys Ther 1998; 78:458-469.

5. Dzienkowski RC, Smith KK, Dillow KA, Yucha CB. Cerebral palsy: a comprehensive review. Nurse Practitioner 1996; 21:45-59.

6. Olney SJ, Wright MJ. Cerebral palsy. In Campbell SK (ed). Physical therapy for children. Philadelphia: Saunders, 1995:489-524.

7. Diament A. Encefalopatias crônicas da infância (paralisia cerebral). In Diament A, CypelS (eds). Neurologia infantil.3.Ed. São Paulo: Atheneu, 1996:781-798.

8. Piovesana AMSG. Encefalopatia crônica (paralisia cerebral): etiologia, classificação e tratamento clínico. In Fonseca LF, Pianetti G, Xavier CC (eds). Compêndio de neurologia infantil. Rio de Janeiro: MEDSI, 2002;825-838.

9. http://www.defnet.org.br/pctaq04.htm.

10. Petersen MC, Kube DA, Palmer FB. Classification of developmental delays. Sem Ped Neurol 1998;5:2-14.

11. Palisano R, Rosenbaum P, Walter S, Russell D, Wood E, Galuppi B. Developmental and reliability of a system to classify gross motor function in children with cerebral palsy. Dev Med Child Neurol 1997;39:214-223.

12. Butler C. Outcomes that matter. Dev Med Child Neurol 1995; 37:753754.

13. Pellegrino L. Cerebral palsy: a paradigma for developmental disabilities. Dev Med Child Neurol 1995; 37:834-839.

14. Haley SM, Coster WJ, Ludlow LH, Haltiwanger JT, Andrellow PJ. Pediatric evaluation of disability inventory: development, standardization and administration manual. Boston: New England Medical Center, 1992:1-300.

15. Haley SM, Coster WJ, Ludlow LH, Haltiwanger JT, Andrellow PJ. Inventário de avaliação pediátrica de disfunção: versão brasileira. Tradução e adaptação cultural: Mancini MC. Belo Horizonte: Laboratórios de Atividade e Desenvolvimento Infantil, Departamento de Terapia Ocupacional, Universidade Federal de Minas Gerais, 2000.

16. Magalhães LC, Fisher AG, Bernspang B, Linacre J.M. Cross-cultural assessment of functional ability. Occup Ther J Research 1996; 16:45-63.

17. Linacre JM, Wright BD. A user's guide to bigsteps: Rasch-model computer Program. Chicago: MESA Press, 1993.

18. Wright BD, Stone MH. Best test design. Chicago: MESA Press, 1979.

19. Ynch EW, Hanson MJ. Developing cross-cultural competence. Baltimore: Paul Brookes Publ, 1992.

20. Bee H. A criança em desenvolvimento. 7.Ed. Porto Alegre: Artes Médicas, 1996.

21. Gesell A. A criança de 0 a 5 anos. São Paulo: Martins Fontes, 1985.

22. Gesell A, Ilg FL, Ames LB, Bullis GE. A criança dos 5 aos 10 anos. 5.Ed. Lisboa: Dom Quixote, 1977.

23. Illingworth RS. The developmental of the infant and young child: normal and abnormal. 9.Ed. New York: Churchill Livingstone, 1987.

24. Illingworth RS. The normal child: some problems of the early years and their treatment. 7.Ed. New York: Churchill Livingstone, 1979.

25. Knobloch H, Passamanick B. Diagnóstico do desenvolvimento: avaliação e tratamento do desenvolvimento neuropsicológico no lactente e na criança pequena: o normal e o patológico. 3.Ed. Rio de Janeiro: Atheneu, 1990.

26. Heriza C. Motor development: traditional and comteporary theories. In Contemporary management of motor control problems: proceedings of II STEP Conference. Alexandria: Foundation for Physical Therapy, 1991:99-126.

27. Atwater SW. Should the normal motor developmental sequence be used as a theoretical model in pediatric physical therapy? In Contemporary management of motor control problems: proceedings of II STEP Conference. Alexandria: Foundation for Physical Therapy, 1991:89-93.

28. Brown M, Gordon WA. Impact of impairment on activity patterns of children. Arch Phys Med Rehabil 1987; 68:828-832.

29. Mackie PC, Jessen EC, Jarvis SN. The lifestyle assessment questionnaire: an instrument to measure the impact of disability on the lives of children whit cerebral palsy and their families. Child Care Health Dev 1998; 24:473-486. 